\title{
Electronic structure of $\mathrm{Ga}_{1-x} \mathrm{Cr}_{x} \mathrm{~N}$ and Si-doping effects studied by photoemission and $\mathrm{X}$-ray absorption spectroscopy
}

\author{
G. S. Song, ${ }^{1}$ M. Kobayashi, ${ }^{1}$ J. I. Hwang, ${ }^{1}$ T. Kataoka, ${ }^{1}$ M. Takizawa, ${ }^{1}$ A. Fujimori,${ }^{1,2}$ \\ T. Ohkouchi, ${ }^{2}$ Y. Takeda,${ }^{2}$ T. Okane,${ }^{2}$ Y. Saitoh, ${ }^{2}$ H. Yamagami, ${ }^{2,3}$ F.-H. Chang, ${ }^{4}$ L. Lee, ${ }^{4}$ \\ H.-J. Lin, ${ }^{4}$ D. J. Huang, ${ }^{4}$ C. T. Chen,${ }^{4}$ S. Kimura,${ }^{5}$ M. Funakoshi,${ }^{5}$ S. Hasegawa, ${ }^{5}$ and H. Asahi ${ }^{5}$ \\ ${ }^{1}$ Department of Physics and Department of Complexity Science and Engineering, \\ University of Tokyo, 7-3-1 Hongo, Bunkyo-ku, Tokyo, 113-0033, Japan \\ ${ }^{2}$ Synchrotron Radiation Research Unit, Japan Atomic Energy Agency, Sayo-gun, Hyogo 679-5148, Japan \\ ${ }^{3}$ Department of Physics, Faculty of Science, Kyoto Sangyo University, Kyoto 603-8555, Japan \\ ${ }^{4}$ National Synchrotron Radiation Research Center, Hsinchu 30076, Taiwan \\ ${ }^{5}$ The Institute of Scientific and Industrial Research, \\ Osaka University, 8-1 Mihogaoka, Ibaraki, Osaka 567-004\%, Japan
}

(Dated: November 5, 2018)

\begin{abstract}
The electronic structure of the magnetic semiconductor $\mathrm{Ga}_{1-x} \mathrm{Cr}_{x} \mathrm{~N}$ and the effect of Si doping on it have been investigated by photoemission and soft $\mathrm{x}$-ray absorption spectroscopy. We have confirmed that $\mathrm{Cr}$ in $\mathrm{GaN}$ is predominantly trivalent substituting for $\mathrm{Ga}$, and that $\mathrm{Cr} 3 d$ states appear within the band gap of GaN just above the $\mathrm{N} 2 p$-derived valence-band maximum. As a result of Si doping, downward shifts of the core levels (except for $\mathrm{Cr} 2 p$ ) and the formation of new states near the Fermi level were observed, which we attribute to the upward chemical potential shift and the formation of a small amount of $\mathrm{Cr}^{2+}$ species caused by the electron doping. Possibility of Cr-rich cluster growth by Si doping are discussed based on the spectroscopic and magnetization data.

PACS numbers: 75.50.Pp, 75.30.Hx, 78.70.Dm, 79.60.-i
\end{abstract}

Diluted magnetic semiconductors (DMSs) with Curie temperatures $\left(T_{\mathrm{C}}\right.$ 's) significantly above room temperature are needed for the development of spintronics devices for practical applications. $\mathrm{Ga}_{1-x} \mathrm{Cr}_{x} \mathrm{~N}$ has been predicted theoretically to be ferromagnetic via double exchange (DE) mechanism [1] and indeed ferromagnetism (FM) above room temperature has been observed [2, 3]. $\mathrm{X}$-ray absorption and photoemission studies have revealed strong hybridization between the $\mathrm{Cr} 3 d$ states and the valence $p$ band [4], and the formation of $\mathrm{Cr} 3 d$-derived states within the band gap of GaN [5, 6], which suggested a high potential as an intrinsic DMS. However, high resistivity [7] and the absence of density of states at Fermi level $\left(E_{\mathrm{F}}\right)[5,6]$ have thrown doubt on the DE mechanism of the FM in $\mathrm{Ga}_{1-x} \mathrm{Cr}_{x} \mathrm{~N}$. On the other hand, the substitutional transition-metal (TM)-rich clusters of nanometer sizes embedded in the host semiconductor have been observed in $\mathrm{Zn}_{1-x} \mathrm{Cr}_{x} \mathrm{Te}$ [8] and suggested as another origin of ferromagnetism in DMSs [9]. Such TM-rich clusters have also been observed in other IIIV-based wide-gap DMSs, $\mathrm{Ga}_{1-x} \mathrm{Mn}_{x} \mathrm{~N}$ [10], $\mathrm{In}_{1-x} \mathrm{Cr}_{x} \mathrm{~N}$ [11], and $\mathrm{Ga}_{1-x} \mathrm{Fe}_{x} \mathrm{~N}$ [12].

In order to clarify the mechanism of the FM in $\mathrm{Ga}_{1-x} \mathrm{Cr}_{x} \mathrm{~N}$, whether the $\mathrm{DE}$ mechanism or the Crrich cluster formation, the investigation of the carrierconcentration dependence of the electronic structure together with the magnetic properties is expected to yield essential information as in the case of $\mathrm{N}$ - and I-doped $\mathrm{Zn}_{1-x} \mathrm{Cr}_{x} \mathrm{Te}$ [13, 14]. Recently, Funakoshi et al. [15] reported a successful control of carrier concentration in $\mathrm{Ga}_{1-x} \mathrm{Cr}_{x} \mathrm{~N}$ by doping $\mathrm{Si}$, which is expected to replace $\mathrm{Ga}$ and hence acts as a donor. In this work, we report on photoemission (PES) and x-ray absorption spectroscopy (XAS) studies of Si-doping effect on the electronic structure of $\mathrm{Ga}_{1-x} \mathrm{Cr}_{x} \mathrm{~N}$ samples whose magnetic properties have been characterized. The results indicate that $\mathrm{Si}$ doping causes an upward chemical potential shift and the formation of new states near $E_{\mathrm{F}}$ (presumably derived from $\mathrm{Cr}^{2+}$ ). We suggest that $\mathrm{Cr}$-rich cluster formation may be enhanced by Si doping, and may stabilize FM or superparamagnetism (SPM) in $\mathrm{Ga}_{1-x} \mathrm{Cr}_{x} \mathrm{~N}$. Possible origins of the observed FM or SPM shall be discussed below.

$\mathrm{Ga}_{1-x} \mathrm{Cr}_{x} \mathrm{~N}$ thin films were grown by radio-frequency (rf) plasma-assisted molecular beam epitaxy on $2 \mu \mathrm{m}$ GaN-template substrates which had been grown by metal-organic chemical vapor deposition. Elemental Ga, $\mathrm{Cr}, \mathrm{Si}$, and rf plasma-enhanced $\mathrm{N}_{2}$ were used as sources. After thermally cleaning the substrate surface at $700{ }^{\circ} \mathrm{C}$ for $15 \mathrm{~min}$, a high-temperature GaN buffer layer was grown at $700{ }^{\circ} \mathrm{C}$. Finally, a $\mathrm{Ga}_{0.98} \mathrm{Cr}_{0.02} \mathrm{~N}(\mathrm{GaCrN})$ or Si-doped $\mathrm{Ga}_{0.98} \mathrm{Cr}_{0.02} \mathrm{~N}$ ( $\mathrm{GaCrN}: \mathrm{Si}$ ) layer was grown at a relatively low temperature of $700{ }^{\circ} \mathrm{C}$ in order to increase the Cr solubility. The Ga flux, $\mathrm{N}_{2}$ flow rate, $\mathrm{Cr}$ cell temperature, and $\mathrm{Si}$ cell temperature were set at $1.6 \times 10^{-7}$ Torr, 1.5 SCCM, $980{ }^{\circ} \mathrm{C}$, and $1100{ }^{\circ} \mathrm{C}$, respectively. The nominal carrier concentrations were $1 \times 10^{18}$ and $2 \times 10^{19}$ $/ \mathrm{cm}^{3}$ for $\mathrm{GaCrN}$ and $\mathrm{GaCrN}: \mathrm{Si}$, respectively, and the $\mathrm{Si}$ concentration should be somewhat larger than them. The thickness of the Cr-doped layer was about $19 \mathrm{~nm}$. For comparison, Si-doped GaN without Cr doping (GaN:Si) was also grown by the same procedure. To avoid the oxidation of sample surfaces and to perform the PES and XAS measurements without surface cleaning, $2 \mathrm{~nm}$ - 

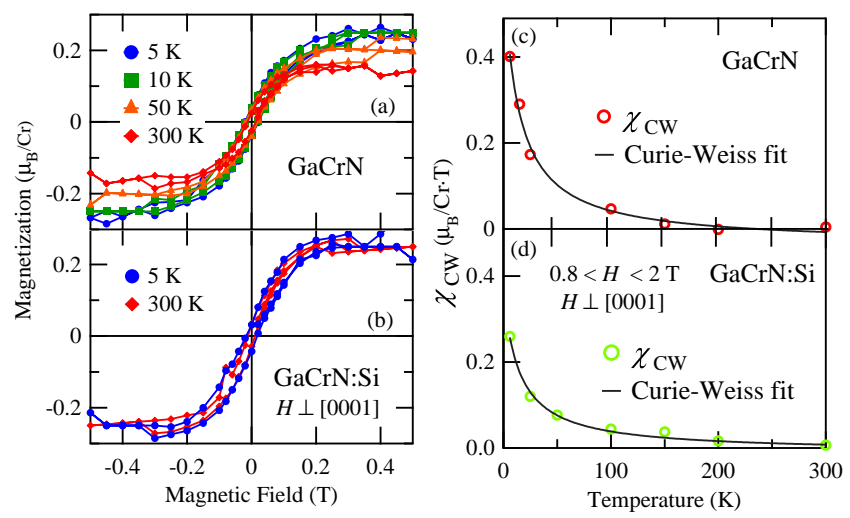

FIG. 1: (Color Online) Magnetization of $\mathrm{GaCrN}$ and GaCrN:Si. (a), (b) $M-H$ curves at various temperatures. (c), (d) Curie-Weiss (CW) component of the high-field magnetic susceptibility as a function of temperature.

thick capping layers ( $\mathrm{GaN}$ for $\mathrm{GaCrN}$ and $\mathrm{GaN}$ :Si for $\mathrm{GaCrN}: \mathrm{Si}$, respectively) were deposited on the sample surfaces. Magnetization was measured using a SQUID magnetometer (MPMS XL, Quantum Design, Co., Ltd.).

$\mathrm{X}$-ray photoemission spectroscopy (XPS) measurements were performed using a $\mathrm{Mg}-K \alpha$ source and a Gammadata Scienta SES-100 hemispherical analyzer. Resonant photoemission spectroscopy (RPES) measurements were performed at BL-23SU of SPring-8 using synchrotron radiation. Photoelectrons were collected using a Gammadata Scienta SES-2002 hemispherical analyzer. The photoemission spectra were referenced to the $\mathrm{Au} 4 f$ peak and the $E_{\mathrm{F}}$ of gold which was in electrical contact with the samples for XPS and RPES, respectively. XAS measurements were performed at the Dragon Beamline BL-11A of National Synchrotron Radiation Research Center in the total-electron-yield mode. All the spectra were taken at room temperature. The total resolution of the spectrometer including the temperature broadening was $\sim 800 \mathrm{meV}$ and $\sim 200 \mathrm{meV}$ for XPS and RPES, respectively. The monochromator resolution for XAS was $E / \Delta E>10,000$. The base pressure of spectrometer was below $3 \times 10^{-8} \mathrm{~Pa}$.

The magnetization data of the two Cr-doped samples are shown in Fig. 11 Panels (a) and (b) shows $M-H$ curves at various temperatures. Here, the linear components have been subtracted. Such a $M-H$ curve is known as an characteristic feature of SPM. One can see that the $T_{\mathrm{C}}$ exceeds $400 \mathrm{~K}$ for both samples. The saturation magnetization of the $\mathrm{GaCrN}$ slightly decreases with increasing temperature, while that of the Si-doped one hardly decreases with temperature, as shown in panels (a) and (b), respectively. Since, in general, saturation magnetization is constant on temperature far below $T_{\mathrm{C}}$, the observation suggests that Si doping in $\mathrm{Ga}_{1-x} \mathrm{Cr}_{x} \mathrm{~N}$ causes an increase of $T_{\mathrm{C}}$. Figure 1(c) and (d) shows the temperature dependence of high-field $(0.8<H<2 \mathrm{~T})$ magnetic susceptibility and Curie-Weiss (CW) fit. The fitting was made assuming a $\mathrm{CW}$ term plus a temperature independent constant $\partial M / \partial H=N C /(T-\Theta)+\chi_{0}$. Here, $N$ is number of the magnetic ions, $g$ is the $g$ factor, $C=\left(g \mu_{B}\right)^{2} S(S+1) / 3 k_{B}$ is the Curie constant (assuming $S=3 / 2$ and $g=2$ ), and $\theta$ is the Weiss temperature. After the fitting, the $\chi_{0}$ term has been removed. Fractions of the $\mathrm{Cr}$ atoms showing a $\mathrm{CW}$-paramagnetic behavior are $\sim 26 \%$ for $\mathrm{GaCrN}$ and $\sim 16 \%$ for $\mathrm{GaCrN}: \mathrm{Si}$. The decrease of the CW component with $\mathrm{Si}$ doping indicates that the number of isolated (paramagnetic) $\mathrm{Cr}$ atoms decreases, probably because they were absorbed by the Cr-rich clusters.

Figure 2(a)-(d) shows the core-level XPS spectra of GaCrN, GaCrN:Si, and GaN:Si. In panel (a), the two peaks in each spectrum are due to the $\mathrm{Cr} 2 p_{3 / 2}-2 p_{1 / 2}$ spin-orbit doublet. The Cr $2 p$ spectrum of $\mathrm{Cr}_{2} \mathrm{O}_{3}\left(\mathrm{Cr}^{3+}\right)$ 17] is also shown for comparison. The peak position for $\mathrm{GaCrN}$ is close to that for $\mathrm{Cr}_{2} \mathrm{O}_{3}$, indicating that the valence of $\mathrm{Cr}$ in $\mathrm{GaCrN}$ is close to 3+, that is, the $\mathrm{Cr}$ atom is doped into $\mathrm{GaN}$ as a neutral impurity if $\mathrm{Cr}$ substitutes for Ga, consistent with the earlier reports [6, 7, 18]. The core-level peaks shown in panels (b)-(d) are shifted downwards in $\mathrm{GaCrN}: \mathrm{Si}$ and $\mathrm{GaN}: \mathrm{Si}$ compared with $\mathrm{GaCrN}$, indicating that the Si doping causes an upward shift of the chemical potential $\mu$. Furthermore, one notices that the core-level peaks of $\mathrm{GaCrN}: \mathrm{Si}$ are shifted upwards compared with $\mathrm{GaN}: \mathrm{Si}$, suggesting that $\mathrm{Cr}$ acts as an acceptor and compensates the electron carriers, consistent with the previous reports that $\mathrm{Cr}$ doping make semicon-

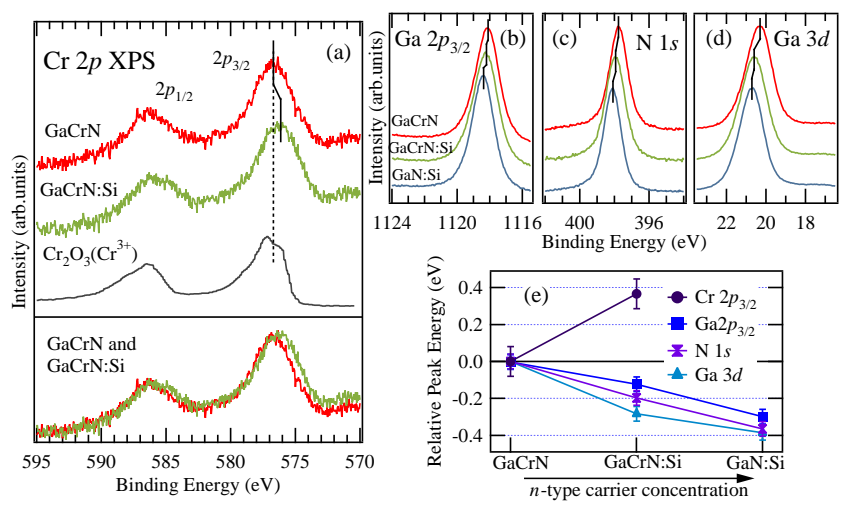

FIG. 2: (Color Online) Core-level XPS spectra of GaCrN, GaCrN:Si and Si-doped GaN (GaN:Si). (a) Cr $2 p$, (b) Ga $3 d$, (c) $\mathrm{N} 1 s$, and (d) Ga $2 p_{3 / 2}$ core levels. Electron mean free paths in $\mathrm{GaP}$ for the $\mathrm{Cr} 2 p, \mathrm{Ga} 3 d, \mathrm{~N} 1 s$, and $\mathrm{Ga} 2 p_{3 / 2}$ core levels are $\sim 2.0, \sim 3.1, \sim 2.3$, and $\sim 0.7 \mathrm{~nm}$, respectively [16]. Vertical lines represent the peak position of each core level. In (a), the Cr $2 p$ core-level XPS spectrum of $\mathrm{Cr}_{2} \mathrm{O}_{3}$ [17] is also shown for comparison. The spectra of GaCrN and GaCrN:Si are overlaid at the bottom panel. (e) Shifts of the core-level peaks of GaCrN:Si and GaN:Si relative to those of GaCrN. The $n$-type carrier concentration increases in order of $\mathrm{GaCrN}$, GaCrN:Si, GaN:Si. 
ducting GaN samples insulating [7, 19]. The amount of the peak shift of each core level relative to that in $\mathrm{GaCrN}$ is plotted in panel (e). The effect of electron trapping by $\mathrm{Cr}$ appears in the $\mathrm{Cr} 2 p$ spectrum of $\mathrm{GaCrN}: \mathrm{Si}$ as shown at the bottom of panel (a): one can see the formation of a small hump for the Si-doped sample and the opposite peak shift to the other core levels. In general, the binding energy $\left(E_{B}\right)$ of a core-level spectrum decreases with decreasing the valence of the element due to the increased screening effect by valence electrons. We therefore consider that electrons supplied by the Si doping are trapped at $\mathrm{Cr}$ site and convert those atoms from $\mathrm{Cr}^{3+}$ to $\mathrm{Cr}^{2+}$.

The Cr $2 p$ XAS spectra of $\mathrm{GaCrN}$ and $\mathrm{GaCrN}: \mathrm{Si}$ are shown in Fig. 3(a). For comparison, those of $\mathrm{Cr}_{2} \mathrm{O}_{3}$ and $\mathrm{Zn}_{1-x} \mathrm{Cr}_{x} \mathrm{Te}$ [20] are also shown. The peak positions and the line shape of the spectrum of $\mathrm{GaCrN}$ agree with those of $\mathrm{Cr}_{2} \mathrm{O}_{3}\left(\mathrm{Cr}^{3+}\right)$, and thus one can conclude that the valence of $\mathrm{Cr}$ in $\mathrm{GaCrN}$ and $\mathrm{GaCrN}: \mathrm{Si}$ is predominantly $3+$. Comparing the spectra of $\mathrm{GaCrN}$ and $\mathrm{GaCrN}: \mathrm{Si}$, one can see a slight difference at $\sim 577 \mathrm{eV}$ and $585 \mathrm{eV}$. The small difference implies that only a small amount of $\mathrm{Cr}^{2+}$ species exist. The difference corresponds well to the overall spectral weight distribution of $\mathrm{Zn}_{1-x} \mathrm{Cr}_{x} \mathrm{Te}\left(\mathrm{Cr}^{2+}\right)$. Figure 3(b) and (c) shows the valence-band RPES spectra of $\mathrm{GaCrN}$ and $\mathrm{GaCrN}: \mathrm{Si}$ recorded in the $\mathrm{Cr} 2 p-3 d$ excitation region. The difference spectra obtained by subtracting the off-resonance spectrum (A) from the onresonance spectra ( $\mathrm{B}$ or $\mathrm{C}$ ) shown at the bottom of panels (b) and (c) represent the $\mathrm{Cr} 3 d$ partial density of states (PDOS). One can clearly see that $\mathrm{Cr} 3 d$-derived emission is seen mostly within the band gap of $\mathrm{GaN}$ as reported in Refs. [5, 6]. Also the existence of Cr-related states at $\sim 340 \mathrm{meV}$ above the valence-band maximum (VBM) was reported in Ref. [19] from a photoluminescence study. The peak position of the Cr $3 d$ PDOS of GaCrN relative to the VBM well agrees with this value. Here, we have estimated the position of the VBM by the intersection between the zero level and a linear extrapolation of the low $E_{B}$ edge of the valence-band spectrum [21]. The distance between the $E_{\mathrm{F}}$ and VBM was estimated to be $\sim 2.9 \mathrm{eV}$ for $\mathrm{GaCrN}$ and $\sim 3.1 \mathrm{eV}$ for GaCrN:Si as shown panels (b) and (c).

Figure 3(d) and (e) shows comparison of the onresonance spectra $(\mathrm{C})$ of the three samples and comparison of the Cr $3 d$ PDOS (C-A) of GaCrN and GaCrN:Si. In panel (e), one clearly finds that new states are formed around $E_{B} \sim 1 \mathrm{eV}$ by the Si doping, as indicated by a vertical arrow. Considering the results of XPS and XAS, we attribute this new feature to a $\mathrm{Cr}^{2+}$ origin. The new states are created as deep as $E_{\mathrm{B}} \sim 1 \mathrm{eV}$ in spite of the small chemical potential shift of $\sim 200 \mathrm{meV}$, quite different from the conventional rigid-band behavior. While the entire valence band is shifted towards higher $E_{B}$ by Si doping, the $\mathrm{Cr} 3 d$ PDOS stays at the same $E_{B}$, as indicated by a vertical dashed line in panel (d). The formation of the new states and the absence of shifts in the Cr $3 d$ PDOS may be explained by the strong Coulomb repulsion between the $\mathrm{Cr} 3 d$ electrons 22]. We note that no $\mathrm{Cr} 3 d$ PDOS was observed at $E_{\mathrm{F}}$. In Fig. $3(\mathrm{f})$, we show a schematic diagram of the electronic structure of $\mathrm{GaCrN}, \mathrm{GaCrN}: \mathrm{Si}$, and GaN:Si where the chemical potential shift, the upward shift of Cr $3 d$ PDOS, and the formation of the new states are presented.

In the preceding paragraphs, we have considered changes in the electronic structure by $\mathrm{Si}$ doping as a result of $\mathrm{Cr}^{2+}$ formation. In order to discuss the relationship between the observed changes in the electronic structure and the magnetism of $\mathrm{Ga}_{1-x} \mathrm{Cr}_{x} \mathrm{~N}$ caused by $\mathrm{Si}$ doping, we consider the formation of Cr-rich clusters as follows. If $\mathrm{Cr}$ ions are randomly distributed in $\mathrm{GaN}$, magnetic interaction between $\mathrm{Cr}$ ions would be weak because the interaction would be short-ranged [23]. Considering the results of the magnetization measurements, which imply the existence of Cr-rich clusters and its growth by $\mathrm{Si}$ doping, and the results of the PES and XAS measure-
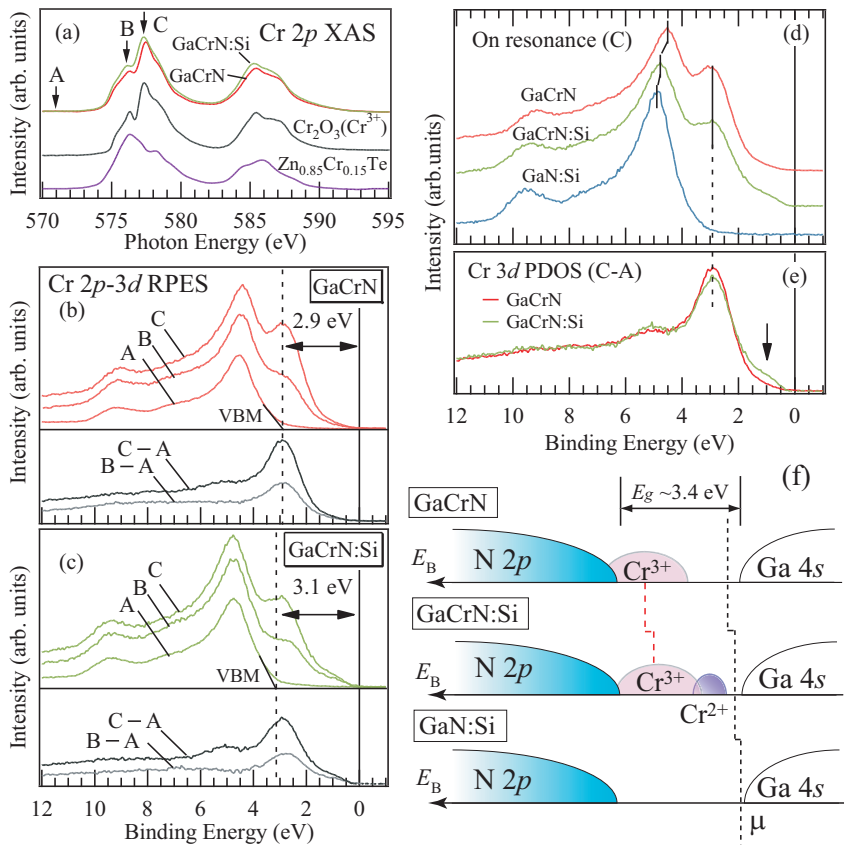

FIG. 3: (Color Online) Cr $2 p$ XAS spectra (a) and Cr $2 p$ $3 d$ RPES spectra of GaCrN (b) and GaCrN:Si (c) taken with photon energies A, B and $\mathrm{C}(h \nu: \mathrm{A} \sim 571.5 \mathrm{eV}, \mathrm{B} \sim 576 \mathrm{eV}, \mathrm{C}$ $\sim 577.5 \mathrm{eV}$ ) as shown in panel (a). The difference spectra B-A and C-A are shown at the bottom of panel (b) and (c). The VBMs estimated from the off-resonance spectra are indicated by vertical dashed lines. (d) Comparison of the on-resonance spectra of the different samples taken with photon energy C. The spectra have been normalized at the peak intensity of the $\mathrm{N} 2 p$-derived valence band at $E_{B} \sim 4.5-5 \mathrm{eV}$. (e) Si-doping dependence of the $\mathrm{Cr} 3 d$ PDOS. Arrow indicates a new $\mathrm{Cr}$ $3 d$ PDOS feature induced by the Si doping. (f) Schematic diagram of the electronic structures of $\mathrm{GaCrN}, \mathrm{GaCrN}: \mathrm{Si}$, and GaN:Si. The shifts of chemical potential $\mu$, the upward shift of $\mathrm{Cr} 3 d$ PDOS peak, and the formation of the new states of $\mathrm{Cr}^{2+}$ origin are shown. Here, the band gap $\sim 3.4 \mathrm{eV}$ of hexagonal GaN is also indicated. 
ments, which suggest the increase of $\mathrm{Cr}^{2+}$ states with Si doping and the absence of Cr $3 d$ PDOS at $E_{\mathrm{F}}$, one can imagine that the coexistence of $\mathrm{Cr}^{3+}$ and $\mathrm{Cr}^{2+}$ ions within the Cr-rich cluster may induce short-range DE within the Cr-rich clusters and stabilize ferromagnetism. However, if there is ferromagnetic spin alignment within the Cr-rich clusters via DE, the saturation magnetization $\left(M_{\mathrm{S}}\right)$ would increase with increasing cluster size significantly. In the present study, we could not observe such an increase of $M_{\mathrm{S}}$ and $M_{\mathrm{S}}$ remained small ( $\sim 0.25$ $\mu_{\mathrm{B}} / \mathrm{Cr}$ ) as shown in Fig. 1(a) and (b). One possible reason for the absence of short-range DE is the insufficient amount of $\mathrm{Cr}^{2+}$. Actually, the concentration of $\mathrm{Si}$ and $\mathrm{Cr}$ is $\sim 10^{19} \mathrm{~cm}^{-3}$ and $\sim 10^{21} \mathrm{~cm}^{-3}$, respectively, and therefore there are only few $\%$ of $\mathrm{Cr}^{3+}$ is converted to $\mathrm{Cr}^{2+}$.

Another scenario to explain the possible increase of $T_{\mathrm{C}}$ by $\mathrm{Si}$ doping is the growth of antiferromagnetic (AFM) Cr-rich clusters. Since the spin moment of the $\mathrm{Cr}^{3+}$ ion is $3 \mu_{\mathrm{B}}, M_{\mathrm{S}} \sim 0.25 \mu_{\mathrm{B}} / \mathrm{Cr}$ means that the amount of ferromagnetic $\mathrm{Cr}$ atoms in $\mathrm{GaCrN}$ and $\mathrm{GaCrN}: \mathrm{Si}$ is only $\sim 8 \%$. The amount of paramagnetic $\mathrm{Cr}$ atoms estimated from the $\mathrm{CW}$ fit is $\sim 26 \%$ and $\sim 16 \%$ for $\mathrm{GaCrN}$ and $\mathrm{GaCrN}: \mathrm{Si}$, respectively. This indicates that $\sim 66 \%$ and $\sim 76 \%$ for $\mathrm{GaCrN}$ and $\mathrm{GaCrN}: \mathrm{Si}$ the $\mathrm{Cr}$ atoms could not be detected by the present magnetization measurements and are probably antiferromagnetically coupled. Recently, Cui et al. 24 have calculated the magnetic moment and the stability of substitutional Cr-rich clusters with several sizes in $\mathrm{Ga}_{1-x} \mathrm{Cr}_{x} \mathrm{~N}$ based on first-principles density functional theory, and have shown that large Crrich clusters are energetically favored and $\mathrm{Cr}$ atoms in these large Cr-rich clusters are coupled antiferromagnetically. The calculated moments of the $\mathrm{Cr}$-rich clusters with various sizes are 0.06-1.47 $\mu_{\mathrm{B}} / \mathrm{Cr}$, corresponding well to the observed $M_{\mathrm{S}} \sim 0.25 \mu_{\mathrm{B}} / \mathrm{Cr}$. Kim et al. 7] have reported that the $M_{\mathrm{S}}$ of $\mathrm{Ga}_{1-x} \mathrm{Cr}_{x} \mathrm{~N}$ decreases with increasing $\mathrm{Cr}$ concentration, which can be interpreted as due to the size increase of the antiferromagnetic Cr-rich clusters. As for $\mathrm{Zn}_{1-x} \mathrm{Co}_{x} \mathrm{O}$, for which even smaller $M_{\mathrm{S}}$ has been reported, Dietl et al. 25] attributed the origin of the small $M_{\mathrm{S}}$ to the uncompensated moments of the Co-rich AFM nanoclusters. These theoretical and ex- perimental reports lead us to speculate that the uncompensated moments from AFM Cr-rich clusters and the increase of the cluster size may be the origin of the observed magnetism and its Si-doping dependence in $\mathrm{Ga}_{1-x} \mathrm{Cr}_{x} \mathrm{~N}$. Also, the magnetic anisotrpy (factor of 2-3 in the saturation magnetization) observed for the present $\mathrm{GaCrN}$ and $\mathrm{GaCrN}: \mathrm{Si}$ samples may be responsible for the observed apparent ferromagnetic benhavior.

We note that the enhancement of FM by donor atom doping is seen in I-doped $\mathrm{Zn}_{1-x} \mathrm{Cr}_{x} \mathrm{Te}$ [8]. In the case of $\mathrm{Zn}_{1-x} \mathrm{Cr}_{x} \mathrm{Te}$, in undoped samples, because $\mathrm{ZnTe}$ is known as a native $p$-type semiconductor, I-doping makes the $\mathrm{Cr}$ ion becomes neutral 2+. Kuroda et al. [8] attributed the origin of the enhancement of FM to the Crrich cluster formation in the I-doped samples caused to attractive forces between neutral $\mathrm{Cr}$ impurities $\left(\mathrm{Cr}^{2+}\right)$ as opposed to the repulsive forces between charged $\mathrm{Cr}$ impurities $\left(\mathrm{Cr}^{3+}\right)$. In the present case, the Si doping makes a fraction of the $\mathrm{Cr}$ ions negatively charged, and therefore the charge neutrality cannot be a driving force of the Crrich cluster formation in the Si-doped GaN. Microscopy observation of $\mathrm{Ga}_{1-x} \mathrm{Cr}_{x} \mathrm{~N}$ :Si are therefore highly desired in future studies.

In summary, we have investigated the Si-doping dependence of the electronic structure of $\mathrm{Ga}_{1-x} \mathrm{Cr}_{x} \mathrm{~N}$ and its relationship to magnetism using XPS, XAS, and RPES. The upward chemical potential shift induced by Si doping into $\mathrm{Ga}_{1-x} \mathrm{Cr}_{x} \mathrm{~N}$ and downward one by $\mathrm{Cr}$ doping into GaN:Si were observed. The new states of $\mathrm{Cr}^{2+}$ character were found to form near $E_{\mathrm{F}}$ in $\mathrm{Cr} 3 d$ PDOS spectra by the $\mathrm{Si}$ doping. The growth of AFM Cr-rich clusters is proposed to explain the observed change of magnetism by the Si doping.

This work was supported by a Grant-in-Aid for Scientific Research in Priority Area gCreation and Control of Spin Currenth(19048012) from MEXT, Japan. The experiment at SPring- 8 was approved by the Japan Synchrotron Radiation Research Institute (JASRI) Proposal Review Committee (Proposal No. 2007A3832). We also thank the Material Design and Characterization Laboratory, Institute for Solid State Physics, University of Tokyo, for the use of the SQUID magnetometer.
[1] K. Sato and H. Katayama-Yoshida, Jpn. J. Appl. Phys. 40, L485 (2001).

[2] M. Hashimoto, Y. K. Zhou, M. Kanamura, H. KatayamaYoshida, and H. Asahi, J. Cryst. Growth 251, 327 (2003).

[3] J. J. Kim, M. Makino, P. P. Chen, T. Suzuki, D. C. Oh, H. J. Ko, J. H. Chang, T. Hanada, and T. Yao, Phys. Stat. Sol. C 0, 2869 (2003).

[4] M. Hashimoto, Y. K. Zhou, M. Kanamura, H. KatayamaYoshida, and H. Asahi, J. Cryst. Growth 273, 149 (2004).

[5] J. J. Kim, H. Makino, K. Yamazaki, A. Ino, H. Namatame, M. Taniguchi, T. Hanada, M. W. Cho, and T. Yao, Curr. Appl. Phys 4, 603 (2004).
[6] J. I. Hwang, Y. Ishida, M. Kobayashi, Y. Osafune, T. Mizokawa, A. Fujimori, Y. Takeda, K. Terai, S.-I. Fujimori, Y. Saitoh, Y. Muramatsu, A. Tanaka, T. Kondo, H. Munekata, M. Hashimoto, H. Tanaka, S. Hasegawa, , and H. Asahi, Phys. Stat. Sol. B 243, 1696 (2006).

[7] J. J. Kim, H. Makino, M. Sakurai, D. C. Oh, T. Hanada, M. W. Cho, T. Yao, S. Emura, and K. Kobayashi, J. Vac. Sci. Technol. B 23, 1308 (2005).

[8] S. Kuroda, N. Nishizawa, K. Takita, M. Mitome, Y. Bando, K. Oshuch, and T. Dietl, Nat. Mater. 6, 440 (2007).

[9] K. Sato, H. Katayama-Yoshida, and P. H. Dederichs, 
Jpn. J. Appl. Phys. 44, L948 (2005).

[10] S. Dhar, O. Brandt, A. Trampert, L. Däweritz, K. J. Friedland, K. H. Ploog, J. Keller, B. Beschoten, and G. Güntherodt, Appl. Phys. Lett. 82, 2077 (2003).

[11] L. Gu, S. Y. Wu, H. Liu, R. K. Singh, N. Newman, and D. J. Smith, J. Magn. Magn. Mater. 290, 1395 (2005).

[12] A. Bonanni, M. Kiecana, C. Simbrunner, T. Li, M. Sawicki, M. Wegscheider, M. Quast, H. Przybylińska, A. Navarro-Quezada, R. Jakiela, A. Wolos, W. Jantsch, and T. Dietl, Phys. Rev. B 75, 125210 (2007).

[13] N. Ozaki, I. Okabayashi, T. Kumekawa, N. Nishizawa, S. Marcet, S. Kuroda, and K. Takita, Appl. Phys. Lett. 87, 192116 (2005).

[14] N. Ozaki, N. Nishizawa, S. Marcet, S. Kuroda, O. Eryu, and K. Takita, Phys. Rev. Lett. 97, 037201 (2006).

[15] M. Funakoshi, Master thesis (Osaka University, 2006).

[16] S. Tanuma, C. J. Powell, and D. R. Penn, Surf. Inter. Analys. 11, 57 (1988).

[17] T. Uozumi, K. Okada, and A. Kotani, J. Electron. Spectrosc. Relat. Phenom. 78, 103 (1996).

[18] M. Hashimoto, S. Emura, H. Tanaka, T. Honma, N. Umesaki, S. Hasegawa, and H. Asahi, J. Appl. Phys. 100,
103907 (2006).

[19] S. Shanthi, S. Kimura, M. S. Kim, S. Kobayashi, Y. K. Zhou, S. Hasegawa, and H. Asahi, Jpn. J. Appl. Phys. 45, 3522 (2006).

[20] M. Kobayashi, Y. Ishida, J. I. Hwang, G. S. Song, A. Fujimori, C.-S. Yang, L. Lee, H.-J. Lin, D.-J. Huang, C. T. Chen, Y. Takeda, K. Terai, S.-I. Fujimori, T. Okane, Y. Saitoh, K. Kobayashi, A. Tanaka, H. Saito, and K. Ando, J. Supercond. Nov. Magn. 20, 467 (2007).

[21] S. A. Chambers, T. Droubay, T. C. Kaspar, and M. Gutowski, J. Vac. Sci. Technol. B 22, 2205 (2004).

[22] K. Morikawa, T. Mizokawa, A. Fujimori, Y. Taguchi, and Y. Tokura, Phys. Rev. B 54, 8446 (1996).

[23] K. Sato, P. H. Dederichs, and H. Katayama-Yoshida, Hyperfine Interactions 160, 57 (2005).

[24] X. Y. Cui, J. E. Medvedeva, B. Delley, A. J. Freeman, N. Newman, and C. Stampfl, Phys. Rev. Lett. 95, 256404 (2005).

[25] T. Dietl, T. Andrearczyk, A. Lipinska, M. Kiecana, M. Tay, and Y. Wu, Phys. Rev. B 76, 155312 (2007). 